\title{
Research on Error Bounds of a Kind of Mixed Variational Inequality Problems
}

\author{
Meimei WANG ${ }^{1}$ and Zhuang SHAN $^{2}$ \\ School of Mathematics and Information Science, North Minzu Universitys, Ningxia, \\ Yinchuan, 750021, China
}

\begin{abstract}
This paper mainly studies mixed variational inequality problems and related problems. We use the residual gap function to represent the regular gap function and $D$-gap function of the mixed variational inequality problem; In addition, we use the regular gap function and the $D$-gap function to describe the error bounds of the mixed variational inequality problem under the assumption of the relaxed cocoercive of the monotonicity operator.
\end{abstract}

Keywords. Variational inequalities, error bounds, gap function.

\section{Introduction}

The mixed variational inequality problem (MVIP) is to find a point $w^{*} \in K$, such that

$$
\left\langle\Phi\left(w^{*}\right), w-w^{*}\right\rangle+g(w)-g\left(w^{*}\right) \geq 0, \forall w \in K
$$

Here, $\langle\cdot \cdot\rangle$ is inner product in $R^{n}, R^{n}$ is a n-dimensional Euclidean space, $g: R^{n} \rightarrow R \cup$ $\{+\infty\}$ is a proper convex function with lower semi-continuous. $K$ is the non-empty closed convex subset of $R^{n}, \Phi: R^{n} \rightarrow R^{n}$ is the continuous mapping.

Lescarret et al. studied MVIP [1,2] for the first time in 1960. With the deepening of research, MVIP has been continuously studied and applied, such as economic equilibrium problem [3]. In 2006, Huang introduced the concept of generalized $g$ projection operator[4] when studying mixed variational inequalities. This operator retains many good properties of projection operators. One of the important tools for inequality problems. $\mathrm{Li}$ [5] used the properties of the generalized $g$-projection operator to express the error bound of the mixed variational inequality problem with the residual gap function under the assumption of strong monotonicity. However, the conclusion is based on the assumption of strong monotonicity operator. Recently, Noor [6] introduced the concept of relaxed cocoercive monotonicity of mapping to study the error bounds of variational inequalities, and relaxed co-coercive monotonicity is a monotonicity that is weaker than strong monotonicity.

This article uses residual gap function to represent regular gap function and $D$-gap function in mixed variational inequality problem; Under the assumption of relaxed co-

\footnotetext{
${ }^{1}$ Corresponding Author, Meimei Wang, School of Mathematics and Information Science,North Minzu Universitys, Ningxia, Yinchuan, 750021, China; E-mail: 596724067@qq.com.

2 Corresponding Author, Zhuang Shan, School of Mathematics and Information Science,North Minzu Universitys, Ningxia, Yinchuan, 750021, China; E-mail: arcsec30@163.com.
} 
coercive monotonicity, the generalized $g$-projection operator is used to express the error bounds of the mixed variational inequality problem with the help of the regular gap function and the $D$-gap function.

\section{Preliminaries}

The necessary definitions and lemmas are given in this chapter. First, assume $K$ is the non-empty closed convex set of $R^{n}, \Phi: R^{n} \rightarrow R^{n}$ is the continuous map. $g: R^{n} \rightarrow R \cup\{+\infty\}$ is a proper convex function with lower semi-continuous.

Definition 1. [5-7] If there is $\beta>0$,

s. t. $\|\Phi(w)-\Phi(v)\| \leq \beta\|w-v\|, \forall w, v \in R^{n}$,

it is said that mapping $\Phi$ is $\beta$ - Lipschitz continuous.

If there is $\tau>0$,

s. t. $\langle\Phi(w)-\Phi(v), w-v\rangle \geq \tau\|\Phi(w)-\Phi(v)\|^{2}, \forall w, v \in R^{n}$,

it is said that mapping $\Phi$ is co-coercive monotone.

If there is $\sigma>0$,

s. t. $\langle\Phi(w)-\Phi(v), w-v\rangle \geq \sigma\|w-v\|^{2}, \forall w, v \in R^{n}$,

it is said that mapping $\Phi$ is $\sigma-$ strongly monotone.

If there is $\psi, \varphi>0$,

s. t. $\langle\Phi(w)-\Phi(v), w-v\rangle \geq-\psi\|\Phi(w)-\Phi(v)\|^{2}+\varphi\|w-v\|^{2}, \forall w, v \in R^{n}$,

it is said that mapping $\Phi$ is $(\psi, \varphi)$ - relaxed co-coercive monotone.

Definition 2. [4] The generalized $g$-distance function $G: R^{n} \times K \rightarrow R \cup\{+\infty\}$ is defined as

$$
G(\xi, u)=\|z\|^{2}-2\langle\xi, z\rangle+\|\xi\|^{2}+2 \gamma g(z), \forall z \in R^{n}, \xi \in K, \gamma>0 .
$$

Definition 3. [4] The generalized $g$-projection operator is defined as

$$
\operatorname{Proj}_{K}^{g}(w)=\{u \in K: G(\xi, u)=\inf \underset{\xi \in K}{G}(\xi, w)\}, \forall w \in R^{n} .
$$

Lemma 4. [4] The following conclusions are hold, if $\gamma>0$, then for any $u \in R^{n}$, $m=\operatorname{Proj}_{K}^{g}(u)$ if and only if

$$
\langle m-u, w-m\rangle+\gamma g(w)-\gamma g(m) \geq 0, \forall w \in K, \gamma>0 .
$$

\section{Error Bound}

This chapter will present the results of this article in the error bounds. First, the residual gap function defined by the generalized $g$-projection[5] is as follows:

$$
R^{\gamma}(z)=z-\operatorname{Proj}_{K}^{g}(z-\gamma \Phi(z)), \gamma>0, z \in R^{n} .
$$


Lemma 5. Let any constant $\gamma>0$, then $R^{\gamma}(z)=0$, if and only if $z \in R^{n}$ is the solution of $M V I P$.

Proof. $R^{\gamma}(z)=0$ is equivalent to $z=\operatorname{Proj}_{K}^{g}(z-\gamma \Phi(z))$. According to lemma 4, we have

$$
\langle\gamma \Phi(z), w-z\rangle+\gamma g(w)-\gamma g(z)=\langle z-(z-\gamma \Phi(z), w-z\rangle+\gamma g(w)-\gamma g(z) \geq 0 .
$$

So, we get

$$
\langle\Phi(z), w-z\rangle+g(w)-g(z) \geq 0
$$

This means $z$ is the solution of MVIP.

Below we introduce the regular gap function under the generalized $g$-projection operator [8], and give some related conclusions and the MVIP error bounds described by the regular gap function. The regular gap function of MVIP as follows:

$$
M^{\gamma}(z)=\max _{w \in R^{n}}\left\{\langle\Phi(z), z-w\rangle+g(z)-g(w)-\frac{1}{2 \gamma}\|z-w\|^{2}\right\}, \forall z \in R^{n}, \gamma>0 .
$$

According to the definition of the regular gap function, we know that for any constant $\gamma>0$, the following is holds

$$
M^{\gamma}(z)=\left\langle\Phi(z), z-\operatorname{Proj}_{K}^{g}(z-\gamma \Phi(z))\right\rangle+g(z)-g\left(\operatorname{Proj}_{K}^{g}(z-\gamma \Phi(z))\right)-\frac{1}{2 \gamma} \| z-\operatorname{Proj}_{K}^{g}\left(z-\gamma \Phi(z) \|^{2}, \forall z \in R^{n} .\right.
$$

Then there is $M^{\gamma}(z) \geq 0$ for any $z \in R^{n}$. Based on Lemma 4, we can obtain the relationship between the residual gap function and the regular gap function.

Lemma 6. For any constant $\gamma>0$, the following is holds

$$
M^{\gamma}(z) \geq \frac{1}{2 \gamma}\left\|R^{\gamma} z\right\|^{2}, \forall z \in R^{n} .
$$

Then $M^{\gamma}(z)=0$ if and only if $z \in R^{n}$ is the solution of MVIP.

Proof. From Lemma 4, we can get

$\left\langle\operatorname{Proj}_{K}^{g}(z-\gamma \Phi(z))-(z-\gamma \Phi(z)), w-\operatorname{Proj}_{K}^{g}(z-\gamma \Phi(z))\right\rangle+\gamma g(w)-\gamma g\left(\operatorname{Proj}_{K}^{g}(z-\gamma \Phi(z))\right) \geq 0 \forall w \in R^{n}$.

Let $w=z$, then we have

$$
\left\langle\Phi(z), z-\operatorname{Proj}_{K}^{g}(z-\gamma \Phi(z))\right\rangle+g(z)-g\left(\operatorname{Proj}_{K}^{g}(z-\gamma \Phi(z)) \geq \frac{1}{\gamma} \| z-\operatorname{Proj}_{K}^{g}\left(z-\gamma \Phi(z)\left\|^{2}=\frac{1}{\gamma}\right\| R^{\gamma} z \|^{2} .\right.\right.
$$

Then, from (2.1) we can get

$$
M^{\gamma}(z)=\left\langle\Phi(z), R^{\gamma}(z)\right\rangle+g(z)-g\left(\operatorname{Proj}_{K}^{g}(z-\gamma \Phi(z))\right)-\frac{1}{2 \gamma}\left\|R^{\gamma}(z)\right\|^{2} \geq \frac{1}{\gamma}\left\|R^{\gamma}(z)\right\|^{2}-\frac{1}{2 \gamma}\left\|R^{\gamma}(z)\right\|^{2}=\frac{1}{2 \gamma}\left\|R^{\gamma}(z)\right\|^{2} .
$$

For any $z \in R^{n}$, if $M^{\gamma}(z)=0$, then there are $R^{\gamma}(z)=0$, so $z$ is the solution of MVIP. On the contrary, let $z$ be the solution of MVIP, then there is $z=\operatorname{Proj}_{K}^{g}(z-\gamma \Phi(z))$, substituting (1), so $M^{\gamma}(z)=0$.

Theorem 7. Assuming the following conditions are true,

(1) $\Phi$ is $\beta$-Lipschitz continuous;

(2) $\Phi$ is $(\psi, \varphi)$-relaxed cocoercive monotone on $R^{n}$ 
If $z^{*}$ is the solution of MVIP, then for any $z \in R^{n}, \gamma>0$ and $\varphi \geq \psi \beta^{2}+\frac{1}{2 \gamma}$, there is

$$
\left\|z-z^{*}\right\| \leq \sqrt{\frac{1}{\varphi-\psi \beta^{2}-\frac{1}{2 \gamma}}} \sqrt{M^{\gamma}(z)}
$$

Proof. Since $z^{*}$ is the solution of MVIP and relaxed co-coercive monotone, we can get

$$
\begin{aligned}
M^{\gamma}(z) & \geq\left\langle\Phi(z), z-z^{*}\right\rangle+g(z)-g\left(z^{*}\right)-\frac{1}{2 \gamma}\left\|z-z^{*}\right\|^{2} \\
& \geq\left\langle\Phi\left(z^{*}\right), z-z^{*}\right\rangle-\psi\left\|\Phi(z)-\Phi\left(z^{*}\right)\right\|^{2}+\varphi\left\|z-z^{*}\right\|^{2}+g(z)-g\left(z^{*}\right)-\frac{1}{2 \gamma}\left\|z-z^{*}\right\|^{2} \\
& \geq\left\langle\Phi\left(z^{*}\right), z-z^{*}\right\rangle-\psi \beta^{2}\left\|z-z^{*}\right\|^{2}+\varphi\left\|z-z^{*}\right\|^{2}+g(z)-g\left(z^{*}\right)-\frac{1}{2 \gamma}\left\|z-z^{*}\right\|^{2} .
\end{aligned}
$$

Therefore, we have

$$
\left\langle\Phi(z), z-z^{*}\right\rangle+g(z)-g\left(z^{*}\right) \geq 0
$$

Combine (2), we have

$$
\begin{aligned}
M^{\gamma}(z) & \geq \varphi-\psi \beta^{2}\left\|z-z^{*}\right\|^{2}+\varphi\left\|z-z^{*}\right\|^{2}-\frac{1}{2 \gamma}\left\|z-z^{*}\right\|^{2} \\
& \geq\left(\varphi-\psi \beta^{2}-\frac{1}{2 \gamma}\right)\left\|z-z^{*}\right\|^{2} .
\end{aligned}
$$

$D$-gap function[8] can be regarded as the difference between two regular gap functions with different parameters. Under some assumptions, the MVIP error bound described by the gap function is given. We define $D$-gap function of MVIP as follows:

$$
\begin{gathered}
H^{\gamma, \lambda}(u)=\left\langle\Phi(z), R^{\gamma}(z)-R^{\lambda}\left(z^{*}\right)\right\rangle+g\left(\operatorname{Proj}_{\mathrm{K}}^{\mathrm{g}}\left(z-\lambda \Phi(z)-g\left(z^{*}\right)\right)\right)-g\left(\operatorname{Proj}_{\mathrm{K}}^{\mathrm{g}}\left(z-\gamma \Phi(z)-g\left(z^{*}\right)\right)\right) \\
+\frac{1}{2 \lambda}\|R(z)\|^{2}-\frac{1}{2 \gamma}\|R(z)\|^{2}, \forall \gamma>\lambda>0, z \in R^{n} .
\end{gathered}
$$

Lemma 8. Let any constant $\gamma>\lambda>0$, the following holds

$\frac{1}{2}\left(\frac{1}{\lambda}-\frac{1}{\gamma}\right)\left\|R^{\lambda} u\right\|^{2}-\frac{1}{2 \gamma}\left\|R^{\gamma}(z)-R^{\lambda}(z)\right\|^{2} \geq H^{\gamma, \lambda}(z) \geq \frac{1}{2}\left(\frac{1}{\lambda}-\frac{1}{\gamma}\right)\left\|R^{\gamma}(z)\right\|^{2}-\frac{1}{2 \lambda}\left\|R^{\gamma}(z)-R^{\lambda}(z)\right\|^{2} \forall z \in R^{n}$, then $H^{\gamma, \lambda}(z)=0$ if and only if $z \in R^{n}$ is the solution of MVIP.

Proof. For all $z \in R^{n}, \gamma>\lambda>0$. By Lemma 4, we get

$$
\begin{aligned}
& \left.\quad \operatorname{Proj}_{K}^{g}(z-\gamma \Phi(z))-(z-\gamma \Phi(z)), w-\operatorname{Proj}_{K}^{g}(z-\gamma \Phi(z))\right\rangle+\gamma g(w)-\gamma g\left(\operatorname{Proj}_{K}^{g}(w-\gamma \Phi(z))\right) \geq 0 . \\
& \text { Let } w=P_{K}^{g}(z-\lambda \Phi(z)) \text {, we get } \\
& \frac{1}{\rho}\left\langle R^{\gamma}(z), R^{\gamma}(z)-R^{\lambda}(z)\right\rangle \geq \rho g\left(\operatorname{Proj}_{K}^{\lambda}(z-\lambda \Phi(z))\right)-\gamma g\left(\operatorname{Proj}_{K}^{g}(z-\gamma \Phi(z))\right)+\left\langle\Phi(z), R^{\gamma}(z)-R^{\lambda}(z)\right\rangle .
\end{aligned}
$$

The combination (3) can be obtained 


$$
\begin{aligned}
H^{\gamma, \lambda}(z) & \geq \frac{1}{\rho}\left\langle R^{\gamma}(z), R^{\gamma}(z)-R^{\lambda}(z)\right\rangle+\frac{1}{2 \lambda}\left\|R^{\lambda}(z)\right\|^{2}-\frac{1}{2 \rho}\left\|R^{\gamma}(z)\right\|^{2} \\
& =\frac{1}{2}\left(\frac{1}{\lambda}-\frac{1}{\gamma}\right)\left\|R^{\lambda}(z)\right\|^{2}+\frac{1}{\gamma}\left\langle R^{\gamma}(z), R^{\gamma}(z)-R^{\lambda}(z)\right\rangle-\frac{1}{2 \gamma}\left\|R^{\gamma}(z)-R^{\lambda}(z)\right\|^{2}-\frac{1}{\gamma}\left\langle R^{\gamma}(z), R^{\gamma}(z)-R^{\lambda}(z)\right\rangle \\
& =\frac{1}{2}\left(\frac{1}{\lambda}-\frac{1}{\gamma}\right)\left\|R^{\lambda}(z)\right\|^{2}-\frac{1}{2 \gamma}\left\|R^{\gamma}(z)-R^{\lambda}(z)\right\|^{2},
\end{aligned}
$$

in the same way

$$
H^{\gamma, \lambda}(z) \leq \frac{1}{2}\left(\frac{1}{\lambda}-\frac{1}{\gamma}\right)\left\|R^{\lambda}(z)\right\|^{2}-\frac{1}{2 \lambda}\left\|R^{\gamma}(z)-R^{\lambda}(z)\right\|^{2} .
$$

By Theorem 7, we know that $z$ is the solution of MVIP when $M^{\gamma}(z)=0$.

Furthermore, we will use the $D$-gap function to represent the MVIP error bound under the assumption of relaxed co-coercive monotone.

Theorem 9. Assuming the following conditions are true :

(1) $\Phi$ is $\beta$-Lipschitz continuous;

(2) $\Phi$ is $(\psi, \varphi)$-relaxed cocoercive monotone on $R^{n}$. If $z^{*}$ is the solution of MVIP. Then for any $z \in R^{n}$ and $\gamma>\lambda>0, \varphi \geq \psi \beta^{2}$, there is

$$
\left\|z-z^{*}\right\| \leq \sqrt{\varphi-\psi \beta^{2}+\frac{1}{2 \lambda}-\frac{1}{2 \gamma}} \sqrt{G^{\prime, \lambda}(z)} .
$$

Proof. According to the $D$-gap map and relaxed cocoercive monotone, we can get

$$
\begin{aligned}
H^{\gamma, \lambda}(z) & =\left\langle F(z), z-z^{*}\right\rangle+g(z)-g\left(z^{*}\right)+\frac{1}{2 \lambda}\left\|z-z^{*}\right\|^{2}-\frac{1}{2 \gamma}\left\|z-z^{*}\right\|^{2} \\
\geq & \geq\left\langle F\left(z^{*}\right), z-z^{*}\right\rangle-\psi\left\|\Phi(z)-\Phi\left(z^{*}\right)\right\|^{2}+\varphi\left\|z-z^{*}\right\|^{2}+g(z)-g\left(z^{*}\right)+\frac{1}{2 \lambda}\left\|z-z^{*}\right\|^{2}-\frac{1}{2 \gamma}\left\|z-z^{*}\right\|^{2} .
\end{aligned}
$$

Since $z^{*}$ is the solution of MVIP, therefore

$$
\left\langle\Phi(z), z-z^{*}\right\rangle+g(z)-g\left(z^{*}\right) \geq 0 .
$$

Combining equation (4), we can get

$$
\begin{aligned}
H^{\gamma, \lambda}(z) & \geq-\psi \beta^{2}\left\|z-z^{*}\right\|^{2}+\varphi\left\|z-z^{*}\right\|^{2}+g(z)-g\left(z^{*}\right)+\frac{1}{2 \lambda}\left\|z-z^{*}\right\|^{2}-\frac{1}{2 \gamma}\left\|z-z^{*}\right\|^{2} \\
& =\left(\varphi-\psi \beta^{2}+\frac{1}{2 \lambda}-\frac{1}{2 \gamma}\right)\left\|z-z^{*}\right\|^{2} .
\end{aligned}
$$

\section{Conclusion}

We use the properties of generalized $g$-projection operators to describe the error bounds of MVIP with different gap mapping under a weaker monotonicity assumption. These works will provide more choices and some theoretical foundations for the study of error bounds of mixed variational inequalities and its algorithm design in the future. 


\section{Funding}

This article was supported by the Postgraduate Innovation Project of North Minzu University (YCX21153).

\section{References}

[1] Lescarret C: Cas d'addition des applications monotones maximales dans un espace de Hilbert[J]. Comptes Rendus de l'Académie Science Paris, 1965, 261(9):1160-1163.

[2] Browder F E: On the unification of the calculus of variations and the theory of monotone nonlinear operators in banach spaces[J]. Proceedings of the National Academy of Sciences, 1966, 56(2):419-425.

[3] Konnov I V,Volotskaya E O: Mixed variational inequalities and economic equilibrium problems[J]. Journal of Applied Mathematics, 2002, 2(6):289-314.

[4] Wu K Q, Huang N J: Properties of the generalized f-projection operator and its applications in Banach spacesp[J]. Nonlinear Analysis Theory Methods and Applications, 2007, 54(1): 399 - 406.

[5] Li X, Zou Y Z: Existence result and error bounds for a new class of inverse mixed quasi-variational inequalities[J]. Journal of Inequalities and Applications, 2016, 2016(1):1-13.

[6] Noor M A:Existence results for quasi variational inequalities[J]. Banach Journal of Mathematical Analysis, 2007, 1(2):186-194.

[7] Kubota K, Fukushima F:Gap function approach to the generalized nash equilibrium problem[J]. Journal of Optimization Theory Applications,2010,144(3):511-531.

[8] Aussel D, Correa M:Gap functions for quasi-variational inequalities and generalized Nash equiblibrium problems[J]. Theory Applications, 2011,151(7): 474-488. 